\title{
Non-linear Soft Tissue Deformations for the Simulation of Percutaneous Surgeries
}

\author{
Jean-Marc Schwartz ${ }^{1}$, Ève Langelier ${ }^{2}$, Christian Moisan ${ }^{3}$, and Denis Laurendeau ${ }^{1}$ \\ ${ }^{1}$ Computer Vision and Systems Laboratory, Univ. Laval, Québec (Qc) G1K 7P4, Canada \\ ${ }^{2}$ Biomechanics Laboratory, Univ. Laval, Québec (Qc) G1K 7P4, Canada \\ 3 iMRI Unit, Quebec City University Hospital, Québec (Qc) G1L 3L5, Canada
}

\begin{abstract}
We introduce a non-linear extension of the tensor-mass method for real-time computation of biological soft tissue deformations. We aim at developing a simulation tool for the planning of cryogenic surgical treatment of liver cancer. This therapy requires careful planning, therefore accurate modeling of the mechanical behavior of organs is required. Our method presently allows real-time computation of non-linear elastic tissue deformations, and further extension towards viscoelasticity modeling is planned.
\end{abstract}

\section{Introduction}

The development of surgery simulation systems requires fast algorithms to allow realtime computation of tissue deformations, as well as accurate modeling of soft tissue mechanical properties. We are currently developing a simulation tool for the planning of percutaneous image-guided cryosurgical treatment of liver cancer. This therapy consists in destroying tumor cells through successive application of freezing and passive thawing cycles [3]. Careful planning is required to optimize the destruction of tumor cells vs. damage to healthy cells. In particular accurate modeling of the geometric, thermal, and mechanical behavior of organs is required.

Several methods have been reported for rapid calculation of linear elastic mechanical deformations, from relatively simple physical models such as the spring-mass model to models based of continuum mechanics such as the Finite Element Method [1]. However experimental characterizations suggest that linear elasticity is only a coarse approximation of real properties of biological soft tissues. Among other studies, Miller et al. [2] identified a viscoelastic constitutive model as accurate for modeling brain tissue deformations.

\section{Non-linear Modeling}

The most promising approach towards real-time computation of non-linear viscoelasticity appeared to be the tensor-mass model introduced by Cotin et al. [1], as it is both time-efficient and physically accurate. It additionally allows local topological changes on mesh elements so that simulation of cutting or perforation is possible. As a first step we show that adequate real-time correction of linear elasticity parameters allows to model different types of non-linear elastic deformations. In our model, expression of force $\boldsymbol{F}_{T_{i}(j)}$ applied on vertex $\boldsymbol{P}_{T_{i}(j)}$ within a tetrahedral mesh element $T_{i}$ is: 


$$
\boldsymbol{F}_{T_{i}(j)}=\sum_{k=0}^{3}\left(\left[\boldsymbol{K}_{j k}^{T_{i}}\right]+\delta \lambda_{i}\left[\boldsymbol{A}_{j k}^{T_{i}}\right]+\delta \mu_{i}\left[\boldsymbol{B}_{j k}^{T_{i}}\right]\right) \boldsymbol{P}_{T_{i}(k)}^{0} \boldsymbol{P}_{T_{i}(k)},
$$

where $\left[\boldsymbol{K}_{j k}^{T_{i}}\right],\left[\boldsymbol{A}_{j k}^{T_{i}}\right]$ and $\left[\boldsymbol{B}_{j k}^{T_{i}}\right]$ are $3 \times 3$ tensors, $\lambda_{i}$ and $\mu_{i}$ are the Lamé coefficients of the material, and $\delta \lambda_{i}$ and $\delta \mu_{i}$ are non-linear corrections. Tensors only depend on the geometry at rest so that precomputation is possible.

Fig. 1a shows two examples of simulated non-linear tissue constitutive laws. Both tissues have stiffness increasing with compression, as it typically occurs with biological samples. Stiffness increases linearly for tissue 1 and stepwise for tissue 2. Fig. 1b shows forces calculated in a simulated perforation of these tissues: in both cases, simulation on a macroscopic mesh correctly follows the non-linear constitutive law.

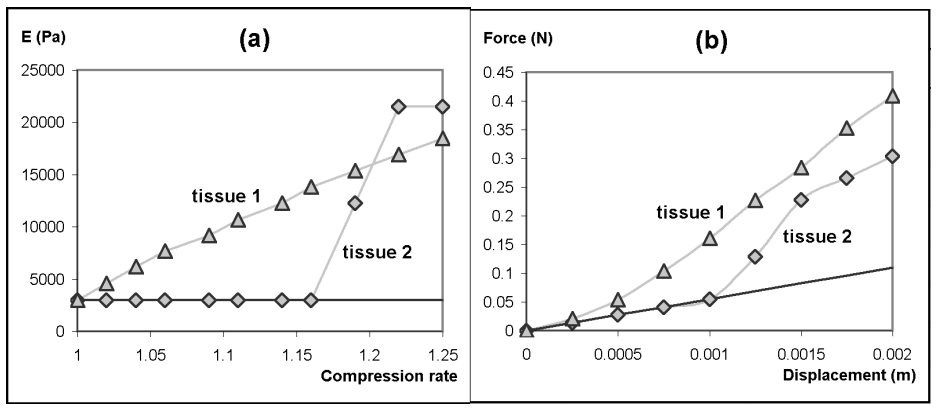

Fig. 1. Two examples of non-linear deformation laws. (a) Young's modulus as a function of the rate of compression. (b) Corresponding force as a function of displacement in simulated perforation. Dark lines represent the linear elastic case for $E=3000 \mathrm{~Pa}$. For all simulations $v=0.4$.

The linear tensor-mass method can typically deal with meshes of a few thousand nodes in real-time. The non-linear extension increases computing time by a factor 4 . However the method remains suitable for real-time applications, provided that the non-linear computational overhead is restricted to a limited number of mesh elements where the highest deformation rates occur.

SEQFurther plans include taking into account viscous effects to allow accurate modeling of biological soft tissues. In addition an experimental setup is under construction to derive experimental data from biological samples in order to assess the accuracy of the model.

\section{References}

1. Cotin, S., Delingette, H., Ayache, N.: A Hybrid Elastic Model for Real-Time Cutting, Deformations, and Force Feedback for Surgery Training and Simulation. Visual Computer 16 (2000) 437-452

2. Miller, K., Chinzei, K., Orssengo, G., Bednarz, P.: Mechanical Properties of Brain Tissue invivo: Experiment and Computer Simulation. J. Biomech. 33 (2000) 1369-1376

3. Morin, J., Dionne, G., Dumont, M., Fouquet, B., Dufour, M., Cloutier, S., Moisan, C.: MR Guided Percutaneous Cryosurgery of Breast Carcinoma: Technique and Early Clinical Results, Proc. Int. Soc. Mag. Res. in Med. (2000) 71 